\title{
Evaluation of low dose of intrathecal morphine in the postoperative pain management of patients undergoing total knee arthroplasty.
}

Acevedo $\mathrm{I}^{*}$., Palomero $\mathrm{M}^{*}$., Dominguez $\mathrm{F}^{*}$., Martin $\mathrm{J}^{*}$., Rodriguez I. ${ }^{*}$

*Anestesiology Department. Ramón y Cajal Universitary Hospital. Madrid. Spain.

\begin{abstract}
Intrathecal morphine administration was a common practice in the previous years. However, this practice is actually discarded due to the adverse effects. The aim of this study is to present our experience with low doses of spinal morphine in the postoperative management of total knee arthroplasty.
\end{abstract}

\begin{abstract}
Background and Goal:
Intrathecal opioid administration was a common practice in the previous years. However, this practice is actually discarded due to the adverse effects. Various studies show that intrathecal morphine used at low doses, has low incidence of adverse effects and provide good control of acute postoperative pain.

The aim of this study is to present our experience with low doses of spinal morphine in the postoperative management of total knee arthroplasty (TKA).
\end{abstract}

\section{Materials and Methods:}

We retrospectively reviewed the records of 27 patients (19 female /8 male), Undergoing TKA under spinal anesthesia with morphine and bupivacaine. The characteristics in terms of age and American Society of Anesthesiologists scale were similar in patients enrolled. All patients were carried to the Postoperative Acute care Unit and discharged between 6 and 10 hours.

In the postoperative period patients were followed by the Acute Pain Management Unit (APMU). Pain assessment by Visual Analogue Scale (VAS), anti-inflammatory medication, numbers of intravenous morphine rescues and side effects were evaluated at 24 and 48 hours.

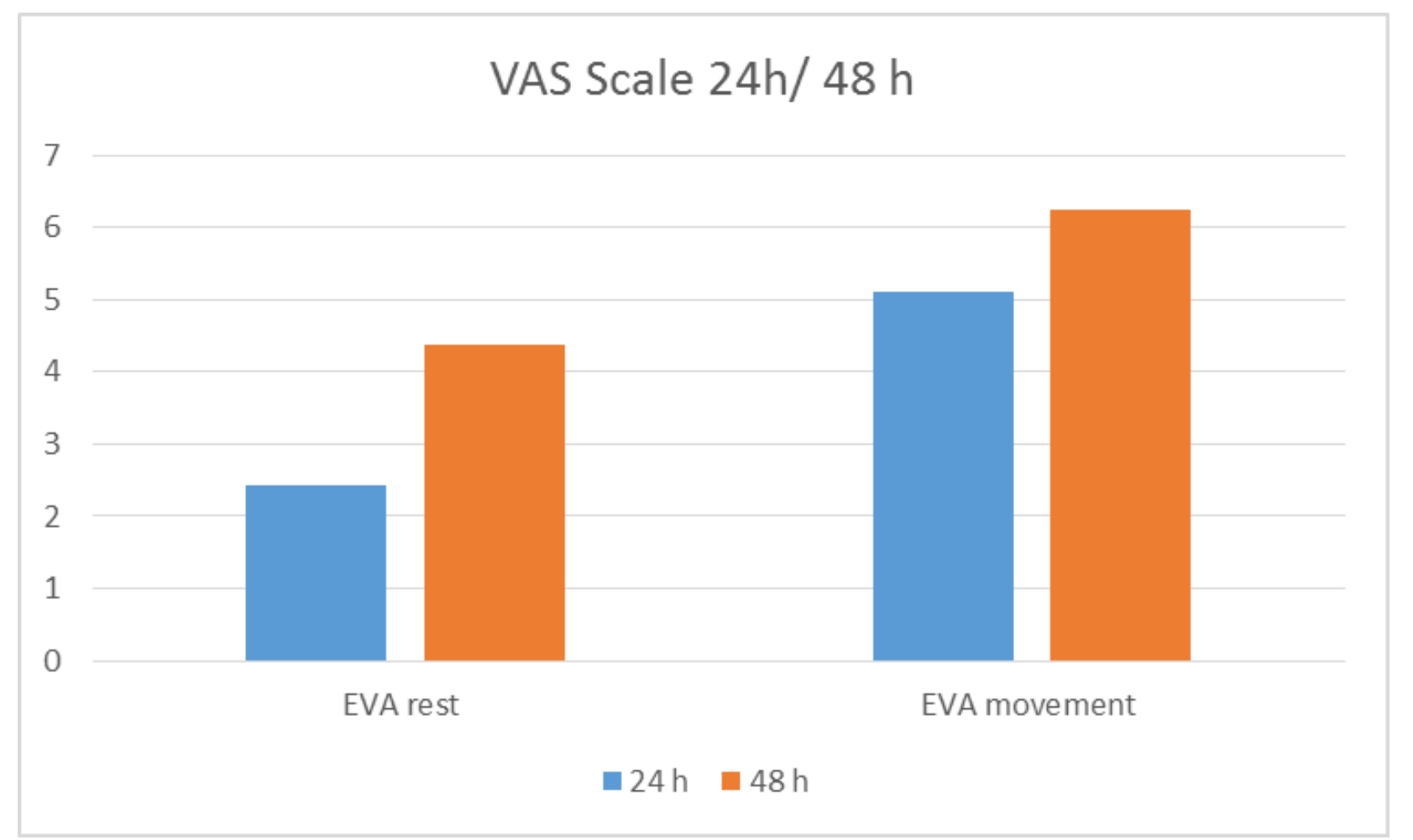

Results and Discussion:

Mean age was 70 years (80/65). American Society Anaesthesiologists score was II in 15 patients and III in 12. Intrathecal morphine was used in combination with hyperbaric bupivacaine $0.5 \%$ (average $8.86 \mathrm{mg}$ ). In 20 patients morphine dose was $70 \mathrm{mcg}$, and in 7 was $100 \mathrm{mcg}$ (average $82.87 \mathrm{mcg}$ ). In all of them the same pattern of postoperative analgesia was followed using the AMPU intravenous protocols for TKA (Dexketoprofen $50 \mathrm{mg}$ each 8 hours combined with Paracetamol $1 \mathrm{~g} / 8 \mathrm{~h}$ and and intravenous morphine rescue with a patient controlled analgesia (PCA) pump, programmed with a bolus of $1 \mathrm{mg}$ each 10 min). The VAS scale average at 24 and 48 hours after surgery, at rest and in motion was 2.43 and $5.1(24 \mathrm{~h}) ; 4.37$ and $6.25(48 \mathrm{~h})$, respectively. Intravenous morphine with PCA requirements average at 24 and 48 was $8.7 \mathrm{mg}$ and $6.71 \mathrm{mg}$, respectively. Two patients had nausea and vomiting, one patient had itching and no one respiratory depression.

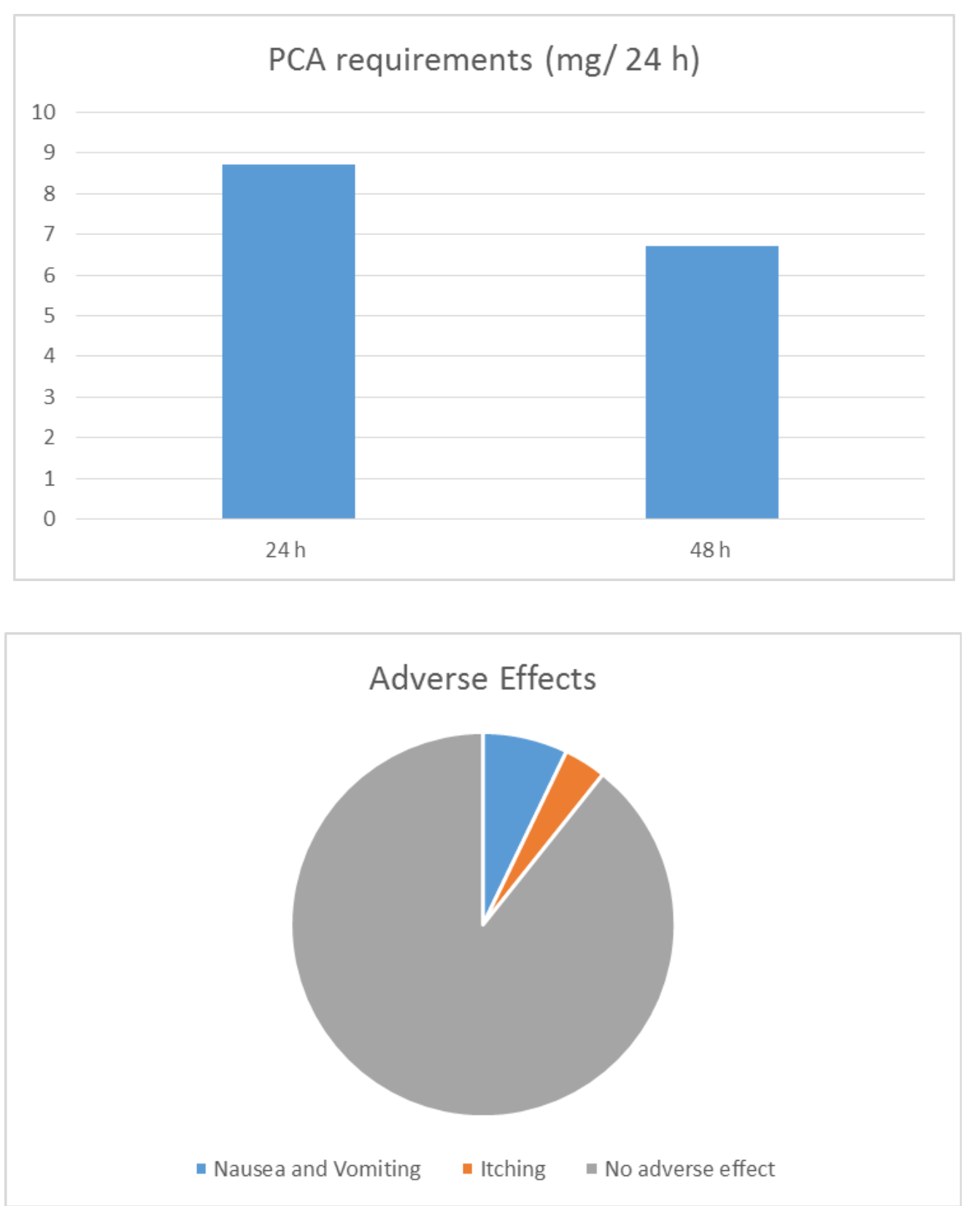

Conclusion:

Intrathecal morphine in a low dose range of $70-100 \mathrm{mcg}$ provides safe and effective postoperative analgesia in the immediate postoperative period for patients with knee arthrosis undergoing total knee replacement. Higher doses do not increase the analgesic effect and can increase the incidence of adverse effects. 